Elect. Comm. in Probab. 16 (2011), 283-295

\title{
QUANTIZATION BALLS AND ASYMPTOTICS OF QUANTIZATION RADII FOR PROBABILITY DISTRIBUTIONS WITH RADIAL EXPO- NENTIAL TAILS
}

\author{
STEFAN JUNGLEN \\ University of Trier \\ email: jung4502@uni-trier.de
}

Submitted February 20th, 2010, accepted in final form April 8th, 2011

AMS 2000 Subject classification: 60E99

Keywords: Maximal Radius, Quantization Radius, Quantization Ball, $(r, r+v)$-distribution

\begin{abstract}
In this paper, we provide the sharp asymptotics for the quantization radius (maximal radius) for a sequence of optimal quantizers for random variables $X$ in $\left(\mathbb{R}^{d},\|\cdot\|\right)$ with radial exponential tails. This result sharpens and generalizes the results developed for the quantization radius in [4] for $d \geq 2$, where the weak asymptotics is established for similar distributions in the Euclidean case. Furthermore, we introduce quantization balls, which provide a more general way to describe the asymptotic geometric structure of optimal codebooks, and extend the terminology of the quantization radius.
\end{abstract}

\section{Introduction and results}

We consider $\left(\mathbb{R}^{d},\|\cdot\|\right)$ equipped with an arbitrary norm and a random variable $X$ in $\mathbb{R}^{d}$ such that for some $r>0$ the $r$-th moment $\mathbb{E}\|X\|^{r}$ is finite. For some natural number $n \in \mathbb{N}$, the quantization problem consists in finding a set $\alpha=\left\{a_{1}, \ldots, a_{n}\right\}$ that minimizes

$$
e_{r}\left(X,\left(\mathbb{R}^{d},\|\cdot\|\right), \alpha\right)=e_{r}\left(X, \mathbb{R}^{d}, \alpha\right):=\left(\mathbb{E} \min _{1 \leq i \leq n}\left\|X-a_{i}\right\|^{r}\right)^{\frac{1}{r}}
$$

over all subsets $\alpha \subset \mathbb{R}^{d}$ with $\operatorname{card}(\alpha) \leq n$. Such sets $\alpha$ are called $n$-codebooks or $n$-quantizers. The corresponding number

$$
e_{n, r}\left(X,\left(\mathbb{R}^{d},\|\cdot\|\right)\right)=e_{n, r}\left(X, \mathbb{R}^{d}\right):=\inf _{\alpha \subset E, \operatorname{card}(\alpha) \leq n} e_{r}\left(X, \mathbb{R}^{d}, \alpha\right)
$$

is called the $n$-th $L^{r}$-quantization error of $X$ in $\mathbb{R}^{d}$, an $n$-quantizer $\alpha$ fulfilling

$$
e_{r}\left(X, \mathbb{R}^{d}, \alpha\right)=e_{n, r}\left(X, \mathbb{R}^{d}\right)
$$

is called $r$-optimal n-quantizer. In the present setting, the existence of optimal quantizers is guaranteed (see [1, Lemma 4.10]). For a given $n$-quantizer $\alpha$, the nearest neighbor projection is given 
by

$$
\pi_{\alpha}: \mathbb{R}^{d} \rightarrow \alpha, \quad x \mapsto \sum_{i=1}^{n} a \mathbb{1}_{C_{a}(\alpha)}(x),
$$

where the Voronoi partition $\left\{C_{a}(\alpha), a \in \alpha\right\}$ is defined as a Borel partition of $\mathbb{R}^{d}$ satisfying

$$
C_{a}(\alpha) \subset V_{a}(\alpha):=\left\{x \in \mathbb{R}^{d}:\|x-a\|=\min _{b \in \alpha}\|x-b\|\right\} .
$$

The random variable $\pi_{\alpha}(X)$ is called the $\alpha$-quantization of $X$. One easily verifies that $\pi_{\alpha}(X)$ is the best quantization of $X$ in $\alpha \subset \mathbb{R}^{d}$, that is for every random variable $Y$ with values in $\alpha$ we have

$$
e_{r}\left(X, \mathbb{R}^{d}, \alpha\right)=\left(\mathbb{E}\left\|X-\pi_{\alpha}(X)\right\|^{r}\right)^{\frac{1}{r}} \leq\left(\mathbb{E}\|X-Y\|^{r}\right)^{\frac{1}{r}} .
$$

One typically is interested in the behavior of $e_{n, r}\left(X, \mathbb{R}^{d}\right)$ when $n$ tends to infinity. By finiteness of $\mathbb{E}\|X\|^{r}$, we deduce that $e_{n, r}\left(X, \mathbb{R}^{d}\right)$ is finite as well. Considering the dense and countable subset $\left\{q_{1}, q_{2}, \ldots\right\}=\mathbb{Q}^{d} \subset \mathbb{R}^{d}$, we estimate

$$
0 \leq \lim _{n \rightarrow \infty} \mathbb{E} \min _{1 \leq i \leq n}\left\|X-q_{i}\right\|^{r}=\mathbb{E} \lim _{n \rightarrow \infty} \min _{1 \leq i \leq n}\left\|X-q_{i}\right\|^{r}=0,
$$

and derive that $e_{n, r}\left(X, \mathbb{R}^{d}\right)$ tends to zero as $n$ tends to infinity.

Naturally, one may now ask for a more precise description of this convergence. To answer this question, it will be convenient to write $a_{n} \sim b_{n}$ for sequences $\left(a_{n}\right)_{n \in \mathbb{N}}$ and $\left(b_{n}\right)_{n \in \mathbb{N}}$ if $\frac{a_{n}}{b_{n}} \stackrel{n \rightarrow \infty}{\longrightarrow} 1$, $a_{n} \lesssim b_{n}$ if $\limsup _{n \rightarrow \infty} \frac{a_{n}}{b_{n}} \leq 1$ and $a_{n} \approx b_{n}$ if $0<\liminf _{n \rightarrow \infty} \frac{a_{n}}{b_{n}} \leq \limsup \sup _{n \rightarrow \infty} \frac{a_{n}}{b_{n}}<\infty$.

Let $\mathbb{E}\|X\|^{r+\delta}<\infty$ for some $\delta>0$ and $h$ be the non-vanishing Lebesgue-continuous part of the density of the distribution $\mathbb{P}^{X}$. Then, the sharp asymptotics of the quantization error is given by

$$
e_{n, r}\left(X, \mathbb{R}^{d}\right) \sim n^{-\frac{1}{d}} Q_{r}(d)^{\frac{1}{r}}\left(\int_{\mathbb{R}^{d}} h^{\frac{d}{d+r}}(x) d \lambda(x)\right)^{\frac{d+r}{r d}}, \quad n \rightarrow \infty .
$$

The numbers $Q_{r}(d) \in(0, \infty)$, depending on $r, d$ and $\|\cdot\|$, are usually unknown, unless in some special cases, for example $d=1,2$. The corresponding quantization coefficient $Q_{r}\left(\mathbb{P}^{X}\right) \in(0, \infty)$ is defined as the limit

$$
Q_{r}\left(\mathbb{P}^{X}\right)=\lim _{n \rightarrow \infty} e_{n, r}^{r}\left(X, \mathbb{R}^{d}\right) n^{\frac{r}{d}} .
$$

This result is known as the Zador Theorem. Its final proof was completed by Graf and Luschgy and can be found in [1, Chapter 6].

As good as the asymptotics of the quantization error can be estimated, as difficult it is to describe the geometric structure of optimal codebooks, or to give at least some asymptotic results on this. For a random variable $X$ with an unbounded support, one easily sees that any sequence $\alpha_{n}$ of $n$-codebooks satisfying

$$
e_{r}\left(X, \mathbb{R}^{d}, \alpha_{n}\right) \stackrel{n \rightarrow \infty}{\longrightarrow} 0
$$

must also fulfill

$$
\rho\left(\alpha_{n}\right):=\max \left\{\|a\|: a \in \alpha_{n}\right\} \stackrel{n \rightarrow \infty}{\longrightarrow} \infty .
$$

Again, one may ask to describe this behavior more precisely, e.g. for sequences $\left(\alpha_{n}\right)_{n \in \mathbb{N}}$ of $r$ optimal $n$-quantizers for $X$. Pagès and Sagna [4] investigated this behavior (they called $\rho_{n}:=$ 
Quantization Balls and Asymptotics of Quantization Radii for Probability Distributions with Radial Exponential Tails

$\rho\left(\alpha_{n}\right)$ the maximal radius) for central symmetric (with respect to the Euclidean norm) distributions with exponential and polynomial tails in the Euclidean $\mathbb{R}^{d}$. For $d=1$ and $r \geq 1$ they derived the sharp asymptotics for distributions with exponential tails. They also gave the weak asymptotics, in case $d \geq 2$ or $r<1$, as well as the sharp logarithmic asymptotics of the maximal radius given polynomial tails. Finally, they formulated a conjecture for the true sharp rate for distributions with radial exponential tails.

In this paper, we proof this conjecture and extend the results for distributions having radial exponential tails to distributions having the density

$$
f(x)=\frac{1}{K}\|x\|_{0}^{c} \exp \left(-\theta\|x\|_{0}^{k}\right), \quad x \in \mathbb{R}^{d} .
$$

Furthermore, we provide the sharp asymptotics of the quantization radius (which will be our terminology for the maximal radius) of a sequence of $r$-optimal $n$-quantizers in $\left(\mathbb{R}^{d},\|\cdot\|\right)$, for arbitrary norms $\|\cdot\|,\|\cdot\|_{0}$ and arbitrary positive $r$. This result extends the formulation of the conjecture to arbitrary norms as well as cases in which the quantizing norm $\|\cdot\|$ does not coincide with the norm $\|\cdot\|_{0}$ in equation (1.3). The latter extension seems to be more important since it includes, for example, normal distributions having a regular non-unit covariance matrix. Furthermore, we introduce quantization balls which provide a more general way to describe the asymptotic geometric structure of optimal codebooks and extend the terminology of the quantization radius.

The paper is organized as follows: In section 2 we introduce the basic notations and provide some technical support for the following section. In section 3 we formulate the main theorem and give the proof for the lower an upper bound (section 3.1 and 3.2). We also transfer the result to the important example of a general normal distribution in $\left(\mathbb{R}^{d},\|\cdot\|_{2}\right)$. In section 4 we provide some numerical illustrations of the results.

\section{Notations:}

- Throughout this paper, we consider a probability space $(\Omega, \mathscr{F}, \mathbb{P}),\left(\mathbb{R}^{d},\|\cdot\|\right)$ for some $d \in \mathbb{N}$ equipped with arbitrary norm $\|\cdot\|$ and a Borel random variable $X$ in $\left(\mathbb{R}^{d},\|\cdot\|\right)$ with finite $r$-th moment $\mathbb{E}\|X\|^{r}<\infty$ for some $r>0$.

- For an arbitrary norm $\|\cdot\|_{0}$ and any $s>0$ we set $B_{\|\cdot\|_{0}}(x, s):=\left\{y \in \mathbb{R}^{d}:\|y-x\|_{0}<s\right\}$.

- For an arbitrary norm $\|\cdot\|_{0}$ we will denote by $\operatorname{dist}_{\|\cdot\|_{0}}(\cdot, \cdot): \mathbb{R}^{d} \times \mathbb{R}^{d} \rightarrow[0, \infty)$ the distance function with respect to the norm $\|\cdot\|_{0}$.

\section{Basics and definitions}

The notations $B(0,1)=B_{\|\cdot\|}(0,1)$ and $\operatorname{dist}(\cdot, \cdot)=\operatorname{dist}_{\|\cdot\| \|}(\cdot, \cdot)$ will be used throughout this section.

Definition 2.1. 1. For a finite codebook $\alpha \subset \mathbb{R}^{d}$ we define the quantization radius by

$$
\rho(\alpha):=\max \{\|a\| \text { with } a \in \alpha\} .
$$

2. Let $\left(\alpha_{n}\right)_{n \in \mathbb{N}}$ be a sequence of $r$-optimal $n$-quantizers for the random variable $X$ in $\left(\mathbb{R}^{d},\|\cdot\|\right)$. We call a subset $\mathscr{B}=\mathscr{B}\left(X, r, \alpha_{n}\right)$ a quantization ball for $X$ in $\left(\mathbb{R}^{d},\|\cdot\|\right)$ of order $r$ if

$$
\mathscr{B}=\overline{\liminf _{n \rightarrow \infty} \frac{\operatorname{conv}\left(\alpha_{n}\right)}{\rho\left(\alpha_{n}\right)}}=\overline{\limsup _{n \rightarrow \infty} \frac{\operatorname{conv}\left(\alpha_{n}\right)}{\rho\left(\alpha_{n}\right)}},
$$


where conv denotes the convex hull and

$$
\begin{aligned}
\liminf _{n \rightarrow \infty} A_{n} & =\bigcup_{n \in \mathbb{N}} \bigcap_{m \geq n} A_{m}, \\
\limsup _{n \rightarrow \infty} A_{n} & =\bigcap_{n \in \mathbb{N}} \bigcup_{m \geq n} A_{m} .
\end{aligned}
$$

If $\mathscr{B}$ is independent of $\left(\alpha_{n}\right)_{n \in \mathbb{N}}$ we call $\mathscr{B}$ the quantization ball.

3. The survival function for the random variable $X$ is given by

$$
\bar{F}: \mathbb{R}_{+} \rightarrow[0,1], \quad x \mapsto \mathbb{P}(\|X\|>x) .
$$

4. For $s \geq 0$ we define the generalized survival function by

$$
\bar{F}_{s}: \mathbb{R}_{+} \rightarrow\left[0, \mathbb{E}\|X\|^{s}\right], \quad x \mapsto \mathbb{E} \mathbb{1}_{\|X\|>x}\|X\|^{s} .
$$

5. The random variable $X$ is said to have an $(r, r+v)$-distribution in $\left(\mathbb{R}^{d},\|\cdot\|\right)$ if for every sequence of $r$-optimal $n$-quantizers $\left(\alpha_{n}\right)_{n \in \mathbb{N}}$

$$
\liminf _{n \rightarrow \infty} \frac{e_{n, r+v}\left(X, \mathbb{R}^{d}\right)}{e_{r+v}\left(X, \mathbb{R}^{d}, \alpha_{n}\right)}>0 .
$$

Since we will focus on distributions with radial exponential tails in the following, we provide relevant results for the survival functions and the $(r, r+v)$-distribution properties for random variables having this density type.

Lemma 2.2. Let $\mathbb{P}^{X}=f \lambda^{d}$ with $f$ having the shape

$$
f(x)=K^{-1}\|x\|^{c} \exp \left(-\theta\|x\|^{k}\right), x \in \mathbb{R}^{d},
$$

for constants $\theta, k>0, c>-d$ and the norming constant

$$
K:=\int_{\mathbb{R}^{d}}\|x\|^{c} \exp \left(-\theta\|x\|^{k}\right) d \lambda^{d}(x) .
$$

Then, for every $s \geq 0$

$$
\bar{F}_{s}(x) \sim C_{\theta, c, k,\|\cdot\|} x^{s+c+d-k} \exp \left(-\theta x^{k}\right), \quad x \rightarrow \infty,
$$

for some constant $C_{\theta, c, k,\|\cdot\|} \in(0, \infty)$.

Proof. Since $\lambda^{d}(B(0, r))=\lambda^{d}(r B(0,1))=r^{d} \lambda^{d}(B(0,1))$, we have

$$
\left(\lambda^{d}\right)^{\|\cdot\|}(d x)=V_{d,\|\cdot\|} d x^{d-1} \lambda(d x)
$$

with $V_{d,\|\cdot\|}:=\lambda^{d}(B(0,1))$. We obtain

$$
\begin{aligned}
\bar{F}_{s}(x) & =\mathbb{E}\left(\|X\|^{s} 1_{\|X\|>x}\right)=K^{-1} \int_{\|y\|>x}\|y\|^{s+c} \exp \left(-\theta\|y\|^{k}\right) d \lambda^{d}(y) \\
& =K^{-1} d V_{d,\|\cdot\|} \int_{x}^{\infty} u^{d+c+s-1} \exp \left(-\theta u^{k}\right) d \lambda(u) .
\end{aligned}
$$


Quantization Balls and Asymptotics of Quantization Radii for Probability Distributions with Radial Exponential Tails

Integration by parts yields

$$
\bar{F}_{s}(x)=K^{-1} d V_{d,\|\cdot\|} \frac{x^{d+c+s-k}}{\theta k} \exp \left(-\theta x^{k}\right)+x^{d+c+s-k} \exp \left(-\theta x^{k}\right) \mathscr{O}\left(\frac{1}{x^{k}}\right)
$$

and thus the assertion with $C_{\theta, c, k,\|\cdot\| \|}:=\frac{K^{-1} d V_{d,\|\cdot\| \cdot \|}}{\theta k}$.

For the following lemma, see [2, Example 1].

Lemma 2.3. Let $\mathbb{P}^{X}=f \lambda^{d}$ with $f$ having the shape

$$
f(x)=K^{-1}\|x\|_{0}^{c} \exp \left(-\theta\|x\|_{0}^{k}\right), x \in \mathbb{R}^{d},
$$

for constants $\theta, k>0, c>-d$, an arbitrary norm $\|\cdot\|_{0}$ and a norming constant $K$. Then, $X$ has an $(r, r+v)$-distribution in $\left(\mathbb{R}^{d},\|\cdot\|\right)$ for all $v<d$.

The proof of the following result is based on the proof in [4] of a similar result in the Euclidean case.

Proposition 2.4. Assume that $\mathbb{P}^{X} \geq \epsilon_{0} \mathbb{1}_{B\left(x_{0}, r_{0}\right)} \lambda^{d}$ for some $\epsilon_{0}, r_{0}>0$. Then, for any $b<\frac{1}{2}$

$$
C_{r, d, b,\|\cdot\|, X} n^{-\frac{r+d}{d}} \lesssim e_{n, r}^{r}\left(X, \mathbb{R}^{d}\right)-e_{n+1, r}^{r}\left(X, \mathbb{R}^{d}\right), \quad n \rightarrow \infty,
$$

where

$$
C_{r, d, b,\|\cdot\|, X}=\left((1-b)^{r}-b^{r}\right) b^{d} \epsilon_{0}\left(\frac{r_{0}}{2}\right)^{r+d} Q_{r+d}(U(B(0,1))) .
$$

Proof. Let $y \in \overline{B\left(x_{0}, \frac{r_{0}}{2}\right)}$ and define $\delta_{n}=\delta_{n}\left(y, \alpha_{n}\right)=\operatorname{dist}\left(y, \alpha_{n}\right)$ for a sequence of $r$-optimal $n$ quantizers $\alpha_{n}$ for $X$. We set $\beta_{n}:=\alpha_{n} \cup\{y\}$. For any $b<\frac{1}{2}$

$$
\begin{aligned}
e_{n, r}^{r}\left(X, \mathbb{R}^{d}\right)-e_{n+1, r}^{r}\left(X, \mathbb{R}^{d}\right) & \geq \int \operatorname{dist}\left(x, \alpha_{n}\right)^{r} d \mathbb{P}^{X}(x)-\int \operatorname{dist}\left(x, \beta_{n}\right)^{r} d \mathbb{P}^{X}(x) \\
& \geq \int_{B\left(y, \delta_{n} b\right)} \operatorname{dist}\left(x, \alpha_{n}\right)^{r}-\operatorname{dist}\left(x, \beta_{n}\right)^{r} d \mathbb{P}^{X}(x) \\
& \geq \int_{B\left(y, \delta_{n} b\right)}\left(\delta_{n}(1-b)\right)^{r}-\left(\delta_{n} b\right)^{r} d \mathbb{P}^{X}(x) \\
& =\left((1-b)^{r}-b^{r}\right) \delta_{n}^{r} \mathbb{P}^{X}\left(B\left(y, \delta_{n} b\right)\right) \\
& \geq\left((1-b)^{r}-b^{r}\right) \delta_{n}^{r+d} b^{d} \epsilon_{0} V_{d,\|\cdot\|},
\end{aligned}
$$

where $V_{d,\|\cdot\|}:=\lambda^{d}\left(B_{\|\cdot\|}(0,1)\right)$. Integration over $B\left(x_{0}, \frac{r_{0}}{2}\right)$ with respect to $d \lambda^{d}(y)$ yields

$$
\begin{aligned}
e_{n, r}^{r}\left(X, \mathbb{R}^{d}\right) & -e_{n+1, r}^{r}\left(X, \mathbb{R}^{d}\right) \geq \frac{\left((1-b)^{r}-b^{r}\right) b^{d} \epsilon_{0} V_{d,\|\cdot\| \cdot}}{\lambda^{d}\left(B\left(0, \frac{r_{0}}{2}\right)\right)} \int_{B\left(x_{0}, \frac{r_{0}}{2}\right)} \operatorname{dist}\left(y, \alpha_{n}\right)^{r+d} d \lambda^{d}(y) \\
& \geq \frac{\left((1-b)^{r}-b^{r}\right) b^{d} \epsilon_{0}}{\left(\frac{r_{0}}{2}\right)^{d}} \int_{B\left(x_{0}, 1\right)} \operatorname{dist}\left(z, \frac{2 \alpha_{n}}{r_{0}}\right)^{r+d}\left(\frac{r_{0}}{2}\right)^{2 d+r} d \lambda^{d}(z) \\
& \geq\left((1-b)^{r}-b^{r}\right) b^{d} \epsilon_{0}\left(\frac{r_{0}}{2}\right)^{d+r} e_{n, r+d}^{r+d}\left(U(B(0,1)), \mathbb{R}^{d}\right),
\end{aligned}
$$

with $U(B(0,1))$ denoting the uniform distribution on $B(0,1)$. The assertion follows in view of the Zador Theorem. 


\section{The main theorem}

For this section, let $X$ be a random variable in $\left(\mathbb{R}^{d},\|\cdot\|\right)$ with $\mathbb{P}^{X}=f \lambda^{d}$ and $f$ having the shape

$$
f(x)=g\left(\|x\|_{0}\right):=K^{-1}\|x\|_{0}^{c} \exp \left(-\theta\|x\|_{0}^{k}\right), x \in \mathbb{R}^{d},
$$

for constants $\theta, k>0, c>-d$, an arbitrary norm $\|\cdot\|_{0}$ and a norming constant $K$. Note, that $g:[0, \infty) \rightarrow[0, \infty]$ has a global supremum in $y^{*}:=\left(\frac{\max (c, 0)}{\theta k}\right)^{\frac{1}{k}}$, which holds as a maximum if $c \geq 0, g$ is increasing on $\left[0, y^{*}\right]$ and decreasing on $\left[y^{*}, \infty\right)$. Furthermore, we set for $n \in \mathbb{N}$

$$
\phi(n)=\phi_{r, d, \theta, k}(n):=\left(\frac{r+d}{\theta d} \log (n)\right)^{\frac{1}{k}} .
$$

Theorem 3.1. For any sequence of $r$-optimal $n$-quantizers $\left(\alpha_{n}\right)_{n \in \mathbb{N}}$ for $X$ in $\left(\mathbb{R}^{d},\|\cdot\|\right)$ it holds

$$
\rho\left(\alpha_{n}\right) \sim \phi(n) \sup _{x \in B_{\|\cdot\|_{0}}(0,1)}\|x\|, \quad n \rightarrow \infty
$$

and the quantization ball $\mathscr{B}$ is given by

$$
\mathscr{B}=\frac{1}{\sup _{x \in B_{\|\cdot\|_{0}}(0,1)}\|x\|} \overline{B_{\|\cdot\|_{0}}(0,1)} .
$$

Remark 3.2. We see that the asymptotics of the quantization radius and the quantization ball are independent of the sequence of quantizers $\left(\alpha_{n}\right)_{n \in \mathbb{N}}$. Furthermore, the quantization ball is independent of the choice of $r$ and, except for a scaling factor, of the underlying norm $\|\cdot\|$.

Example 3.3. We consider $\left(\mathbb{R}^{d},\|\cdot\|\right)=\left(\mathbb{R}^{d},\|\cdot\|_{2}\right)$. Let $\mathbb{P}^{X}$ be a centered $d$-dimensional normal distribution with regular covariance matrix $\Sigma$ and corresponding non increasing ordered eigenvalues $\lambda_{1} \geq \cdots \geq \lambda_{d}>0$. Its density is given by

$$
f(x)=\frac{1}{\sqrt{\left((2 \pi)^{d} \operatorname{det} \Sigma\right)}} \exp \left(-\frac{1}{2}\left\|\Sigma^{-\frac{1}{2}} x\right\|_{2}^{2}\right),
$$

where $\|\cdot\|_{2}$ denotes the Euclidean norm in $\mathbb{R}^{d}$. Thus, it has the form (3.1) with $c=0, \theta=\frac{1}{2}, k=2$ and $\|\cdot\|_{0}=\left\|\Sigma^{-\frac{1}{2}} \cdot\right\|_{2}$. The operator norm of the natural embedding $j:\left(\mathbb{R}^{d},\|\cdot\|_{0}\right) \rightarrow\left(\mathbb{R}^{d},\|\cdot\|_{2}\right)$ is given as the root of the biggest eigenvalue $\lambda_{1}$ of the covariance matrix. Using Theorem 3.1 we obtain the asymptotics of the quantization radius for any sequence of $r$-optimal $n$-quantizers $\left(\alpha_{n}\right)_{n \in \mathbb{N}}$ for $X \stackrel{d}{=} N(0, \Sigma)$ of order $r$ as

$$
\rho\left(\alpha_{n}\right) \sim \sqrt{\lambda_{1}} \phi_{r, d, \frac{1}{2}, 2}(n), \quad n \rightarrow \infty
$$

and the quantization ball is given as the normalized unit ball in $\left(\mathbb{R}^{d},\|\cdot\|_{0}\right)$

$$
\frac{1}{\sqrt{\lambda_{1}}} B_{\left\|\Sigma^{-\frac{1}{2}} \cdot\right\|_{2}}(0,1)
$$

The proof of Theorem 3.1 consists of two parts, one for the lower and one for the upper bound. For convenience we will denote by $C \in[1, \infty)$ the smallest constant satisfying $\frac{1}{C}\|\cdot\| \leq\|\cdot\|_{0} \leq C\|\cdot\|$. 
Quantization Balls and Asymptotics of Quantization Radii for Probability Distributions with Radial Exponential Tails

\subsection{Lower bound}

Proposition 3.4. Let $\left(\alpha_{n}\right)_{n \in \mathbb{N}}$ be a sequence of $r$-optimal n-quantizers for the random variable $X$ in $\left(\mathbb{R}^{d},\|\cdot\|\right)$. Then, for every $\delta \in(0,1)$ and every sequence $\left(b_{n}\right)_{n \in \mathbb{N}} \in \overline{B_{\|\cdot\|_{0}}(0,1-\delta)}$

$$
\underset{\|\cdot\|}{\operatorname{dist}}\left(\alpha_{n}, b_{n} \phi(n)\right) \rightarrow 0, \quad n \rightarrow \infty .
$$

Proof. Assume that there is a $\delta \in(0,1)$ and a sequence $\left(b_{n}\right)_{n \in \mathbb{N}} \in \overline{B_{\|\cdot\|_{0}}(0,1-\delta)}$ such that (3.2) does not hold. Then, there exists an $\epsilon>0$ and a strictly increasing subsequence $\left(n_{j}\right)_{j \in \mathbb{N}} \in \mathbb{N}^{\mathbb{N}}$ satisfying

$$
B_{\|\cdot\|}\left(b_{n_{j}} \phi\left(n_{j}\right), \epsilon\right) \cap \alpha_{n_{j}}=\emptyset .
$$

We fix $0<v<d$. Using (3.3) we obtain

$$
\begin{aligned}
\mathbb{E}\left\|X-\pi_{\alpha_{n_{j}}}(X)\right\|^{r+v} & \geq \int_{B_{\|\cdot\| \cdot \|}\left(b_{n_{j}} \phi\left(n_{j}\right), \epsilon / 2\right)} \min _{a \in \alpha_{n_{j}}}\|X-a\|^{r+v} d \mathbb{P} \\
& \geq\left(\frac{\epsilon}{2}\right)^{r+v} \mathbb{P}\left(X \in B_{\|\cdot\|}\left(b_{n_{j}} \phi\left(n_{j}\right), \frac{\epsilon}{2}\right)\right) \\
& \geq\left(\frac{\epsilon}{2}\right)^{r+v} \mathbb{P}\left(X \in B_{\|\cdot\|_{0}}\left(b_{n_{j}} \phi\left(n_{j}\right), \frac{\epsilon}{2 C}\right)\right) .
\end{aligned}
$$

Case 1: There is a subsequence of $\left(n_{j}\right)_{j \in \mathbb{N}}$, for convenience also denoted $\left(n_{j}\right)_{j \in \mathbb{N}}$, such that $\left\|b_{n_{j}} \phi\left(n_{j}\right)\right\|_{0}-\frac{\epsilon}{2 C} \geq y^{*}$ for all $j \in \mathbb{N}$. Then, by monotonicity of $g$ and (3.4)

$$
\mathbb{E}\left\|X-\pi_{\alpha_{n_{j}}}(X)\right\|^{r+v} \geq\left(\frac{\epsilon}{2}\right)^{r+v} \lambda^{d}\left(B_{\|\cdot\|_{0}}\left(0, \frac{\epsilon}{2 C}\right)\right) g\left(\left\|b_{n_{j}} \phi\left(n_{j}\right)\right\|_{0}+\frac{\epsilon}{2 C}\right) .
$$

Taking the $(r+v)$-th root and the negative logarithm, we obtain by using the $(r, r+v)$-distribution property

$$
\frac{1}{d} \log \left(n_{j}\right) \lesssim \frac{\theta\left(\left\|b_{n_{j}} \phi\left(n_{j}\right)\right\|_{0}+\frac{\epsilon}{2 C}\right)^{k}}{r+v} \lesssim \frac{\theta(1-\delta)^{k}}{r+v} \frac{(r+d)}{\theta d} \log \left(n_{j}\right), \quad j \rightarrow \infty,
$$

which yields a contradiction by letting $v$ go to $d$.

Case 2: There is an $N \in \mathbb{N}$ such that $\left\|b_{n_{j}} \phi\left(n_{j}\right)\right\|_{0}-\frac{\epsilon}{2 C}<y^{*}$ for all $j \geq N$. Therefore, with $A:=B_{\|\cdot\|_{0}}\left(0, y^{*}+\frac{\epsilon}{C}\right) \backslash B_{\|\cdot\|_{0}}\left(0, \frac{\epsilon}{4 C}\right)$ and (3.4)

$$
\begin{aligned}
\mathbb{E}\left\|X-\pi_{\alpha_{n_{j}}}(X)\right\|^{r+v} & \geq\left(\frac{\epsilon}{2}\right)^{r+v} \lambda^{d}\left(B_{\|\cdot\|_{0}}\left(b_{n_{j}} \phi\left(n_{j}\right), \frac{\epsilon}{2 C}\right) \backslash B_{\|\cdot\|_{0}}\left(0, \frac{\epsilon}{4 C}\right)\right) \inf _{x \in A} f(x) \\
& \geq\left(\frac{\epsilon}{2}\right)^{r+v} \lambda^{d}\left(B_{\|\cdot\|_{0}}\left(0, \frac{\epsilon}{2 C}\right) \backslash B_{\|\cdot\|_{0}}\left(0, \frac{\epsilon}{4 C}\right)\right) \inf _{x \in A} f(x)
\end{aligned}
$$

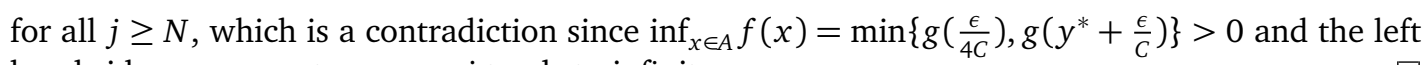
hand side converges to zero as $j$ tends to infinity.

The following corollary provides the lower bound for the main theorem. 
Corollary 3.5. We use the notations from Proposition 3.4. For every $\delta \in(0,1)$ there exists an $n_{\delta} \in \mathbb{N}$ such that

$$
B_{\|\cdot\|_{0}}(0, \phi(n)(1-\delta)) \subset \operatorname{conv}\left(\alpha_{n}\right)
$$

for all $n \geq n_{\delta}$. As an immediate consequence

$$
B_{\|\cdot\|_{0}}(0,1) \subset \overline{\liminf _{n \rightarrow \infty} \frac{\operatorname{conv}\left(\alpha_{n}\right)}{\phi(n)} .}
$$

The lower bound for the quantization radius is given by

$$
\phi(n) \sup _{x \in B_{\|\cdot\| \|_{0}}(0,1)}\|x\| \lesssim \rho\left(\alpha_{n}\right), \quad n \rightarrow \infty .
$$

Proof. Assume that (3.5) does not hold. Then, there exists a strictly increasing sequence $\left(n_{k}\right)_{k \in \mathbb{N}} \in$ $\mathbb{N}^{\mathbb{N}}$ and $z_{n_{k}} \in B_{\|\cdot\|_{0}}\left(0, \phi\left(n_{k}\right)(1-\delta)\right) \backslash \operatorname{conv}\left(\alpha_{n_{k}}\right), k \in \mathbb{N}$. We show that there are $x_{n_{k}} \in \partial B_{\|\cdot\|_{0}}\left(0, \phi\left(n_{k}\right)(1-\right.$ $\left.\frac{\delta}{2}\right)$ ) satisfying

$$
B_{\|\cdot\|_{0}}\left(x_{n_{k}}, \frac{\delta}{2} \phi\left(n_{k}\right)\right) \cap \alpha_{n_{k}}=\emptyset .
$$

By using a separation theorem, we find for every $k \in \mathbb{N}$ a continuous linear $f_{n_{k}}$, such that

$$
f_{n_{k}}\left(z_{n_{k}}\right)>f_{n_{k}}(y) \quad \forall y \in \operatorname{conv}\left(\alpha_{n_{k}}\right) .
$$

Since $\overline{B_{\|\cdot\|_{0}}(0,1)}$ is compact and $f_{n_{k}}$ continuous, there is $x_{n_{k}}^{\prime} \in \overline{B_{\|\cdot\|_{0}}(0,1)}$ such that

$$
f_{n_{k}}\left(x_{n_{k}}^{\prime}\right)=\sup _{y \in \overline{\|_{\|\cdot\|_{0}}(0,1)}} f_{n_{k}}(y) .
$$

We set $x_{n_{k}}:=x_{n_{k}}^{\prime} \phi\left(n_{k}\right)\left(1-\frac{\delta}{2}\right)$. Hence, $x_{n_{k}} \in \partial B_{\|\cdot\|_{0}}\left(0, \phi\left(n_{k}\right)\left(1-\frac{\delta}{2}\right)\right)$ and for $y \in B_{\|\cdot\|_{0}}\left(x_{n_{k}}, \frac{\delta}{2} \phi\left(n_{k}\right)\right)$

$$
\begin{aligned}
f_{n_{k}}(y) & =f_{n_{k}}\left(x_{n_{k}}\right)+f_{n_{k}}\left(y-x_{n_{k}}\right) \geq f_{n_{k}}\left(x_{n_{k}}^{\prime}\right) \phi(n)\left(1-\frac{\delta}{2}\right) \\
& -\sup _{y \in B_{\|\cdot\|_{0}}(0,1)} f_{n_{k}}(y) \phi(n) \frac{\delta}{2}=f_{n_{k}}\left(x_{n_{k}}^{\prime}\right) \phi(n)(1-\delta) \geq f_{n_{k}}\left(z_{n_{k}}\right),
\end{aligned}
$$

and thus

$$
B_{\|\cdot\|_{0}}\left(x_{n_{k}}, \frac{\delta}{2} \phi\left(n_{k}\right)\right) \cap \alpha_{n_{k}}=\emptyset .
$$

Equivalence of the norms implies

$$
B_{\|\cdot\|}\left(x_{n_{k}}, \frac{\delta}{2 C} \phi\left(n_{k}\right)\right) \cap \alpha_{n_{k}}=\emptyset,
$$

which is a contradiction to Proposition 3.4. (3.6) follows by (3.5) and

$$
B_{\|\cdot\|_{0}}(0,1)=\bigcup_{\delta>0} B_{\|\cdot\|_{0}}(0,(1-\delta)) \subset \overline{\liminf _{n \rightarrow \infty} \frac{\operatorname{conv}\left(\alpha_{n}\right)}{\phi(n)}} .
$$

By continuity of $\|\cdot\|_{0}$ we choose $x^{*} \in \overline{B_{\|\cdot\|_{0}}(0,1)}$ such that $\left\|x^{*}\right\|=\sup _{y \in \overline{B_{\|\cdot\|_{0}}(0,1)}}\|y\|$. By (3.5) it follows for $\delta \in(0,1)$

and thus (3.7).

$$
\left\|x^{*}\right\| \phi(n)(1-\delta) \lesssim \rho\left(\alpha_{n}\right), \quad n \rightarrow \infty
$$


Quantization Balls and Asymptotics of Quantization Radii for Probability Distributions with Radial Exponential Tails

\subsection{Upper bound}

Proposition 3.6. Let $\left(\alpha_{n}\right)_{n \in \mathbb{N}}$ be a sequence of $r$-optimal n-quantizers for $X$ in $\left(\mathbb{R}^{d},\|\cdot\|\right)$. Then, for every $\delta>0$ there exists an $n_{\delta} \in \mathbb{N}$ such that

$$
\alpha_{n} \subset B_{\|\cdot\|_{0}}(0, \phi(n)(1+\delta))
$$

for all $n \geq n_{\delta}$.

Proof. Assume that there is a strictly increasing subsequence $\left(n_{k}\right)_{k \in \mathbb{N}} \in \mathbb{N}^{\mathbb{N}}$ with

$$
a_{n_{k}} \in \alpha_{n_{k}} \cap B_{\|\cdot\|_{0}}\left(0, \phi\left(n_{k}\right)(1+\delta)\right)^{c} .
$$

Step 1: We show that

$$
V_{a_{n_{k}}}\left(\alpha_{n_{k}}\right) \subset B_{\|\cdot\|_{0}}\left(0, \phi\left(n_{k}\right)(1+\epsilon)\right)^{c}
$$

for some $\epsilon>0, k_{0} \in \mathbb{N}$ and all $k \geq k_{0}$. With (3.9), we obtain

$$
\underset{\|\cdot\|_{0}}{\operatorname{dist}}\left(a_{n_{k}}, B_{\|\cdot\|_{0}}\left(0, \phi\left(n_{k}\right)\left(1+\frac{\delta}{2}\right)\right)\right) \geq \frac{\delta}{2} \phi\left(n_{k}\right)
$$

which implies in view of equivalence of the norms

$$
\underset{\|\cdot\|}{\operatorname{dist}}\left(a_{n_{k}}, B_{\|\cdot\|_{0}}\left(0, \phi\left(n_{k}\right)\left(1+\frac{\delta}{2}\right)\right)\right) \geq \frac{\delta}{2 C} \phi\left(n_{k}\right) .
$$

On the other hand, it holds with $\epsilon:=\frac{\delta}{12 C^{2}}$

$$
\underset{\|\cdot\|}{\operatorname{dist}}\left(\alpha_{n_{k}} \backslash\left\{a_{n_{k}}\right\}, x_{n_{k}}\right) \leq \frac{\delta}{4 C} \phi\left(n_{k}\right)
$$

for all sequences $x_{n_{k}} \in B_{\|\cdot\|_{0}}\left(0, \phi\left(n_{k}\right)(1+\epsilon)\right)$ since, by choosing $b_{n_{k}}:=x_{n_{k}} \frac{1-\epsilon}{1+\epsilon}$ in Proposition 3.4, there exists a $k_{0} \in \mathbb{N}$ such that

$$
\underset{\|\cdot\|}{\operatorname{dist}}\left(\alpha_{n_{k}}, b_{n_{k}}\right) \leq \epsilon \phi\left(n_{k}\right), \quad \forall k \geq k_{0},
$$

which leads for every $k \geq k_{0}$ to

$$
\begin{aligned}
\underset{\|\cdot\|}{\operatorname{dist}}\left(\alpha_{n_{k}}, x_{n_{k}}\right) & \leq \operatorname{dist}\left(\alpha_{n_{k}}, b_{n_{k}}\right)+\left\|b_{n_{k}}-x_{n_{k}}\right\| \\
& \leq \frac{\delta}{12 C^{2}} \phi\left(n_{k}\right)+\left|\frac{1-\epsilon}{1+\epsilon}-1\right|\left\|x_{n_{k}}\right\| \\
& \leq \frac{\delta}{12 C} \phi\left(n_{k}\right)+\left|\frac{1-\epsilon}{1+\epsilon}-1\right| C\left\|x_{n_{k}}\right\|_{0} \\
& \leq \frac{\delta}{12 C} \phi\left(n_{k}\right)+2 \epsilon C \phi\left(n_{k}\right) \\
& \leq \frac{\delta}{4 C} \phi\left(n_{k}\right) .
\end{aligned}
$$

Combining (3.10) and (3.11) we obtain

$$
V_{a_{n_{k}}}\left(\alpha_{n_{k}}\right) \subset B_{\|\cdot\|_{0}}\left(0, \phi\left(n_{k}\right)(1+\epsilon)\right)^{c}
$$


for all $k \geq k_{0}$.

Step 2: By choosing in Proposition $3.4 b_{n}=0$ for $n \in \mathbb{N}$, we find a sequence $c_{n_{k}} \in \alpha_{n_{k}}$ with $\epsilon_{k}:=\left\|c_{n_{k}}\right\| \rightarrow 0$. By using (3.12) and Proposition 2.4, we get for some constant $K>0$

$$
\begin{aligned}
K n_{k}^{-\frac{d+r}{d}} & \lesssim e_{n_{k}-1, r}^{r}(X, E)-e_{n_{k}, r}^{r}(X, E) \\
& \leq \mathbb{E}\left\|X-f_{\alpha_{n_{k}} \backslash\left\{a_{n_{k}}\right\}}(X)\right\|^{r}-\mathbb{E}\left\|X-f_{\alpha_{n_{k}}}(X)\right\|^{r} \\
& \leq \int_{X \in V_{a_{n_{k}}}\left(\alpha_{n_{k}}\right)}\left\|X-c_{n_{k}}\right\|^{r} d \mathbb{P} \\
& \leq \int_{X \in B_{\|\cdot\|_{0}}\left(0, \phi\left(n_{k}\right)(1+\epsilon)\right)^{c}} 2^{r}\left(\|X\|^{r}+\left\|c_{n_{k}}\right\|^{r}\right) d \mathbb{P} \\
& \leq \int_{X \in B_{\|\cdot\|_{0}}\left(0, \phi\left(n_{k}\right)(1+\epsilon)\right)^{c}} 2^{r} C\|X\|_{0}^{r} d \mathbb{P}+\int_{X \in B_{\|\cdot\|_{0}}\left(0, \phi\left(n_{k}\right)(1+\epsilon)\right)^{c}} 2^{r} \epsilon_{k}^{r} d \mathbb{P},
\end{aligned}
$$

as $k \rightarrow \infty$. Applying Lemma 2.2 with $s=0$ and $s=r$ leads to

$$
\begin{aligned}
K n_{k}^{-\frac{d+r}{d}} & \lesssim 2^{r} C_{\theta, c, k,\|\cdot \cdot\|_{0}}\left(\phi\left(n_{k}\right)(1+\epsilon)\right)^{d+c-k} \times \\
& \exp \left(-\theta \phi\left(n_{k}\right)^{k}(1+\epsilon)^{k}\right)\left(C\left(\phi\left(n_{k}\right)(1+\epsilon)\right)^{r}+\epsilon_{k}^{r}\right),
\end{aligned}
$$

as $k \rightarrow \infty$. Finally, taking the negative logarithm we get

$$
\theta\left(\phi\left(n_{k}\right)(1+\epsilon)\right)^{k} \lesssim \frac{d+r}{d} \log \left(n_{k}\right), \quad k \rightarrow \infty .
$$

This is equivalent to

$$
(1+\epsilon)^{k} \leq 1
$$

which yields a contradiction.

The following corollary provides the upper bound for the main theorem. It follows from Proposition 3.6, the definition of lim sup and a similar argumentation as for (3.6) and (3.7).

Corollary 3.7. We use the notations from Proposition 3.6. For every $\delta>0$ exists an $n_{\delta} \in \mathbb{N}$ such that

$$
\alpha_{n} \subset \operatorname{conv}\left(\alpha_{n}\right) \subset B_{\|\cdot\|_{0}}(0, \phi(n)(1+\delta))
$$

for all $n \geq n_{\delta}$. This immediately leads to

$$
\limsup _{n \rightarrow \infty} \frac{\operatorname{conv}\left(\alpha_{n}\right)}{\phi(n)} \subset \overline{B_{\|\cdot\|_{0}}(0,1)} .
$$

The upper bound for the quantization radius is given by

$$
\rho\left(\alpha_{n}\right) \lesssim \phi(n) \sup _{x \in B_{\|\cdot\|_{0}}(0,1)}\|x\|, \quad n \rightarrow \infty .
$$


Quantization Balls and Asymptotics of Quantization Radii for Probability Distributions with Radial Exponential Tails

Proof of Theorem 3.1. From equation (3.7) and (3.14) we obtain the asymptotics

$$
\rho\left(\alpha_{n}\right) \sim \phi(n) \sup _{x \in B_{\|\cdot\|_{0}}(0,1)}\|x\|, \quad n \rightarrow \infty .
$$

Then, (3.15), (3.5) and (3.13) yield for every $\delta^{\prime}>0$ an $n_{\delta^{\prime}}$ such that for all $n \geq n_{\delta^{\prime}}$

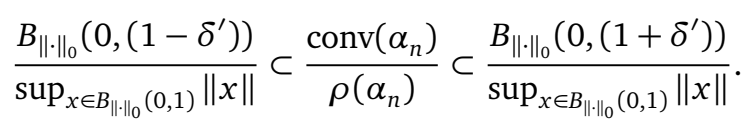

The definition of liminf and limsup,

$$
\bigcup_{\delta>0} B_{\|\cdot\|_{0}}(0,(1-\delta))=B_{\|\cdot\|_{0}}(0,1)
$$

and

$$
\bigcap_{\delta>0} B_{\|\cdot\|_{0}}(0,(1+\delta))=\overline{B_{\|\cdot\|_{0}}(0,1)}=\left\{x:\|x\|_{0} \leq 1\right\}
$$

give the assertion.

\section{Numerical illustration}

Finally, we want to illustrate some of our results. For the computation of the optimal codebooks presented below, we used the CLVQ-Algorithm, see [3]. We consider the Euclidean $\mathbb{R}^{2}, r=2$ and $X \stackrel{d}{=} N(0, \Sigma)$ with eigenvalues $\lambda_{1}=1$ and $\lambda_{2}=\frac{1}{4}$. The figures show the 2-optimal $n$-quantizers for $n=50,250,1000$. The two ellipses in the figures are the scaled quantization balls $\mathscr{B} \rho\left(\alpha_{n}\right)$ and $\mathscr{B} \phi(n)$ with $\phi(n)$ as in section 3.

As already mentioned in [4] for the unit-covariance case, we see that in this case the quantization radius $\rho\left(\alpha_{n}\right)$ seems for finite $n$ to be smaller than its asymptotic equivalent $\phi(n)$ as well.

Furthermore, we observe that for small $n$ the convex hull of $\alpha_{n}$ does not completely fill the ellipse $\mathscr{B} \rho\left(\alpha_{n}\right)$, whereas for growing $n$ almost the whole ellipse seems to be filled by $\operatorname{conv}\left(\alpha_{n}\right)$. 


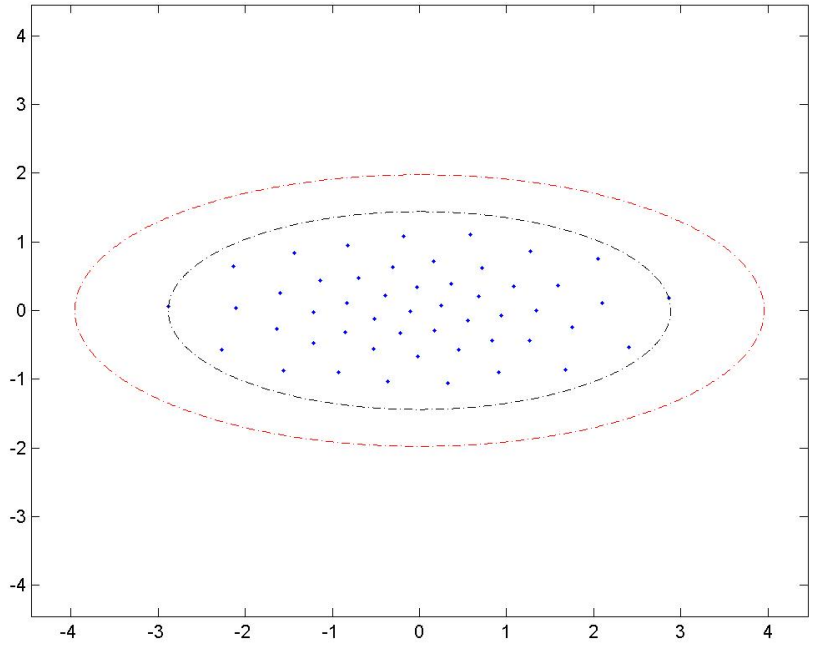

Figure 1: 2-optimal 50-quantizer for $X \stackrel{d}{=} N(0, \Sigma)$, eigenvalues $\lambda_{1}=1$ and $\lambda_{2}=\frac{1}{4},\|\cdot\|=\|\cdot\|_{2}$

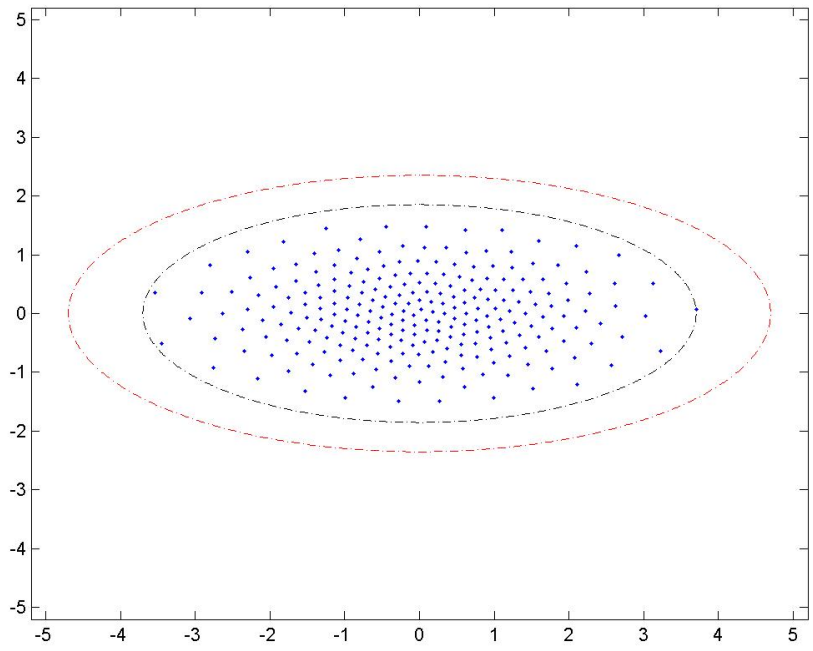

Figure 2: 2-optimal 250-quantizer for $X \stackrel{d}{=} N(0, \Sigma)$, eigenvalues $\lambda_{1}=1$ and $\lambda_{2}=\frac{1}{4},\|\cdot\|=\|\cdot\|_{2}$ 
Quantization Balls and Asymptotics of Quantization Radii for Probability Distributions with

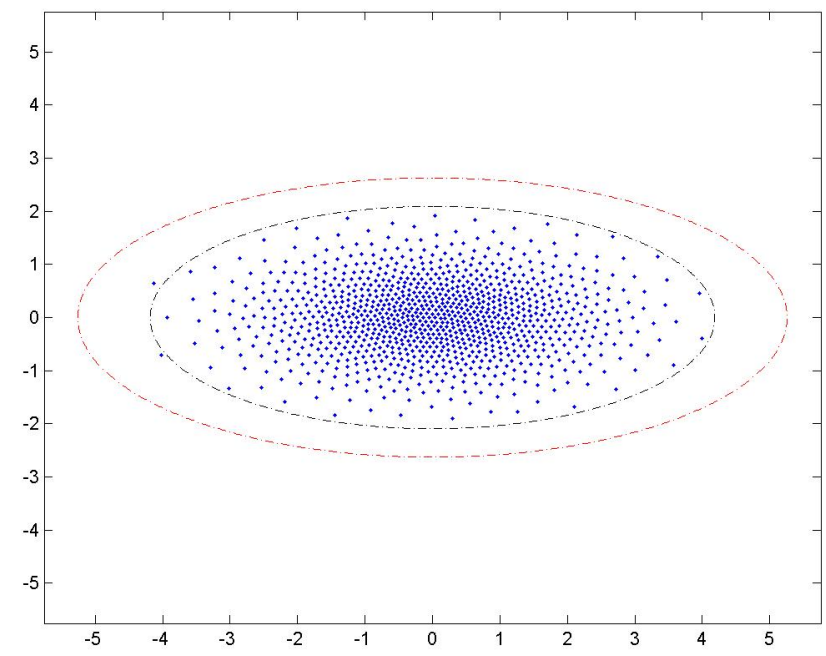

Figure 3: 2-optimal 1000-quantizer for $X \stackrel{d}{=} N(0, \Sigma)$, eigenvalues $\lambda_{1}=1$ and $\lambda_{2}=\frac{1}{4},\|\cdot\|=\|\cdot\|_{2}$

\section{References}

[1] S. Graf and H. Luschgy. Foundations of Quantization for Probability Distributions. SpringerVerlag Berlin, 2000. MR1764176

[2] S. Graf, H. Luschgy, and G. Pagès. Distortion mismatch in the quantization of probability measures. ESAIM: Probability and Statistics, 12:127 - 153, 2008.

[3] G. Pagès. A space quantization method for numerical integration. Journal of Computational and Applied Mathematics, 89(1):1-38, 1998.

[4] G. Pagès and A. Sagna. Asymptotics of the maximal radius of an $L^{r}$-optimal sequence of quantizers. http://www.citebase.org/abstract?id=oai:arXiv.org:0806.0918, 2008. 